\title{
Oxymorons: a preliminary corpus investigation
}

\author{
Marta La Pietra \\ University of Bologna Alumna \\ marta.lapietra93@gmail.com
}

\author{
Francesca Masini \\ University of Bologna \\ francesca.masini@unibo.it
}

\begin{abstract}
This paper contains a preliminary corpus study of oxymorons, a figure of speech so far under-investigated in NLP-oriented research. The study resulted in a list of 376 oxymorons, identified by extracting a set of antonymous pairs (under various configurations) from corpora of written Italian and by manually checking the results. A complementary method is also envisaged for discovering contextual oxymorons, which are highly relevant for the detection of humor, irony and sarcasm.
\end{abstract}

\section{Why oxymorons?}

The growing body of research on figurative language in NLP has recently witnessed an expansion from more traditional domains (like idioms, metaphors, metonymy) to other ubiquitous phenomena such as irony, puns and sarcasm. However, other - supposedly more marginal - figures of speech have received less attention so far. The oxymoron is a case in point.

The oxymoron, which has been studied mainly in rhetoric and literature, is "[a] figure of speech in which a pair of opposed or markedly contradictory terms are placed in conjunction for emphasis", although its meaning has expanded to comprise more generally "a contradiction in terms" (definitions from OED: www.oed.com). Typical examples of oxymorons would be deafening silence, sweet sorrow or awfully good.

The oxymoron is obviously closely intertwined with the semantic relation of antonymy: it is often the union of antonymous items that creates the oxymoron's paradoxical effect, thus generating a new meaning, which often heavily depends on context. As Gibbs \& Kearney (1994: 86) observe, "[u]nderstanding oxymora requires that people access relevant world knowledge to constrain their creative interpretations of seemingly contradictory concepts".

In this paper, we set out a preliminary investigation of oxymorons based on naturally occurring data from Italian, with a view to contributing to the NLP-oriented research on figurative language by supplying an initial list of oxymorons and oxymoronic structures that can be used for further analyses and for evaluation tasks. The questions that drove our investigation are: What kind of oxymorons do we find in common language? What syntactic constructions are involved in their creation? And above all, how can we detect them in corpora?

In Section 2 we describe the methodology we used to detect oxymorons in corpora of contemporary written Italian. Although our study is based on Italian, the procedure we followed can easily be extended to other languages. In Section 3 we illustrate the main results of our analysis. The full list of oxymorons detected is available in the Appendix. Section 4 outlines another promising complementary methodology to be pursued in future research. Finally, Section 5 discusses possible further developments, with special attention to the challenges oxymorons pose for automatic identification and extraction.

\section{Methodology}

The procedure we devised to track down oxymorons in corpora of written Italian stems from the observation that these constructions are closely connected with antonymous pairs, which have been the subject of several studies based on their co-occurrence in texts (cf. e.g. Charles \& Miller 1989; Justeson \& Katz 1991; Lobanova 2012; Kostić 2017). 
Our starting point was Jones' (2002) analysis of English antonyms, which makes use of a list of canonical antonymous pairs to be searched in a corpus of texts from The Independent (approx. $280 \mathrm{M}$ words). We therefore translated Jones' antonymous pairs into Italian and made a selection out of this set, driven mainly by the exclusion of predicative (e.g. confirm $\sim$ deny) and adverbial (e.g. badly $\sim$ well) couples.

This resulted in a list of 17 noun $\sim$ noun antonymous pairs, displayed in Table 1 .

\begin{tabular}{ll}
\hline $\begin{array}{l}\text { Italian antonymous } \\
\text { pairs }\end{array}$ & English translation \\
\hline amore $\sim$ odio & love $\sim$ hate \\
attività $\sim$ passività & activity $\sim$ passivity \\
caldo $\sim$ freddo & hot $\sim$ cold \\
coraggio $\sim$ paura & bravery $\sim$ fear \\
distanza $\sim$ vicinanza & distance $\sim$ proximity \\
dolcezza $\sim$ amarezza & sweetness $\sim$ bitterness \\
felicità $\sim$ infelicità & happiness $\sim$ unhappiness \\
giustizia $\sim$ ingiustizia & justice $\sim$ injustice \\
guerra $\sim$ pace & war $\sim$ peace \\
leggerezza $\sim$ pesantezza & lightness $\sim$ heaviness \\
lentezza $\sim$ velocità & slowness $\sim$ speed \\
luce $\sim$ buio & light $\sim$ dark \\
realtà $\sim$ irrealtà & reality $\sim$ unreality \\
ricchezza $\sim$ povertà & wealth $\sim$ poverty \\
silenzio $\sim$ rumore & silence $\sim$ noise \\
vita $\sim$ morte & life $\sim$ death \\
vuoto $\sim$ pieno & emptiness $\sim$ fullness \\
\hline
\end{tabular}

Table 1: Starting list of Italian antonymous pairs.

Then we designed an inventory of potential oxymorons. All the constructed couples were searched, as lemmas, in two large corpora of contemporary written Italian: Italian Web 2016 (itTenTen16, through the SketchEngine platform: https://www.sketchengine.eu/) and CORIS (2017 version; Rossini Favretti et al. 2002; http://corpora.ficlit.unibo.it/coris _eng.html). The results were manually checked.

The above-mentioned inventory of potential oxymorons was built in the following way.

First, we matched each noun of each pair (e.g. odio 'hate') with its antonym (amore 'love') in either adjectival (amoroso / amorevole 'loving') or verbal (amare 'to love') form. With this first round of extractions, we obtained combinations such as odio amoroso (lit. hate lovely) and amorevole odio (lit. lovely hate) 'loving hate', as well as l'amore odia 'love hates', although sequences containing verbs were quite uncommon. However, the search for lemma verbs retrieved also a number of participial forms used as adjectives (as in amore odiato 'hated love', where odiato is the past participle of odiare 'to hate').

Second, we added contrastive adjective adverb pairs connected to the nouns in Table 1 (e.g. felicità 'happiness' > felice 'happy' > felicemente 'happily' + infelicità > infelice 'unhappy', gaining felicemente infelice 'happily unhappy').

In addition, to enrich data retrieval, we selected lexemes semantically related to the members of the antonymous pairs in Table 1 (synonyms, hyponyms, etc.) from the Grande Dizionario Analogico della Lingua Italiana (Simone 2010). This step was inspired by Shen's (1987: 109) definition of indirect oxymoron, i.e. an oxymoron where "one of [the] two terms is not the direct antonym of the other, but rather the hyponym of its antonym" (like whistling silence, where whistling is a type of noise). Related lexemes were also searched in the two above-mentioned corpora. For the sake of exemplification, we illustrate some of these paradigmatic expansions for the following three antonymous pairs, for which we retrieved a considerable amount of data:

- caldo $~$ freddo (hot $\sim$ cold) $\rightarrow$ afa 'stuffiness', calura 'heat', fuoco 'fire', fiamma 'flame' gelo 'frost', ghiaccio 'ice', grandine 'hail', neve 'snow', pioggia 'rain';

- felicità $\sim$ infelicità (happiness $\sim$ unhappiness) $\rightarrow$ allegria 'glee', contentezza 'cheer', gaiezza 'gaiety', gioia 'joy' afflizione 'distress', depressione 'depression', disperazione 'despair', dolore 'pain', sconforto 'discouragement', scontento 'discontentment', tristezza 'sadness';

- silenzio $\sim$ rumore (silence $\sim$ noise) $\rightarrow$ [silenzio only] boato 'rumble', fracasso 'racket', fragore 'clamour', grido 'shout', ululato 'howling', urlo 'scream'.

The final phase of the analysis implied the interrogation of the Sketch Engine Word Sketch tool, which describes the collocational behavior of words by showing the lexemes that most typically co-occur with them, within specific syntagmatic contexts, by using statistical association measures. In this case, we searched for all nouns participating in the antonymous pairs in Table 1 and we manually revised all top results provided the Word Sketch function (thus focusing on the most statistically significant combinations). Beside oxymorons that we had 
already retrieved with the previous procedure (e.g. the very frequent silenzio assordante 'deafening silence'), this method allowed us to identify new configurations, for instance sentential patterns where the two opposite nouns are linked by the copula è 'is' (e.g. la luce è tenebra 'light is darkness') or prepositional phrases where the two opposite nouns are linked by a preposition (e.g. il fragore del silenzio 'the racket of silence').

\section{Results}

The multiple-step procedure described in Section 2 resulted in a final list of 376 oxymorons, the first of its kind in Italian, to the best of our knowledge. The full dataset (Italian oxymorons 1.0) is provided in the Appendix and released as an Excel file through the University of Bologna Institutional Research Repository (AMSActa):

http://amsacta.unibo.it/id/eprint/6388

Around $20 \%$ of the oxymorons were found in both corpora, whereas the vast majority (almost $80 \%$ ) was retrieved in itTenTen16, which is much larger than CORIS (4.9 billion vs. 150 million words).

\subsection{Syntactic structure}

We classified the 376 oxymorons according to their syntactic structure. A quantitative summary is given in Table 2, which reports, for each of the 9 structures we could identify, the number of oxymorons with that structure and the number of antonymous pairs that generate oxymorons with that structure.

\begin{tabular}{lrr}
\hline $\begin{array}{l}\text { Syntactic } \\
\text { structure }\end{array}$ & $\begin{array}{r}\text { N. of } \\
\text { oxymorons }\end{array}$ & $\begin{array}{r}\text { N. of } \\
\text { antonymous } \\
\text { pairs }\end{array}$ \\
\hline N A & 140 & 17 \\
A N & 112 & 17 \\
S & 52 & 11 \\
Adv A & 50 & 16 \\
N Prep N & 14 & 7 \\
A A & 3 & 1 \\
V V & 2 & 1 \\
V A & 2 & 1 \\
V Prep N & 1 & 1 \\
\hline Total & $\mathbf{3 7 6}$ & $\mathbf{1 7}$ \\
\hline
\end{tabular}

Table 2: Syntactic structures of oxymorons in our dataset.
As expected, the vast majority of the oxymorons in our dataset belongs to noun-adjective combinations, in both orders: [N A] (e.g. silenzio urlante 'screaming silence', attività passive 'passive activities') and [A N] (e.g. raggiante oscurità 'glowing darkness', disperata felicità 'desperate happiness'). These are the only two structures that host oxymorons generated from all the 17 antonymous pairs in Table 1 . In our data, [A $\mathrm{N}]$ sequences are quite numerous despite the fact that noun-adjective is the unmarked, neutral order in Italian. This may be linked to the fact that the prenominal position for Italian adjectives is generally associated with affect and emphasis (Ramaglia 2010), values that are highly compatible with the oxymoron as a rhetorical device. Several examples in these two classes display participial forms used in adjectival function, e.g. fiamma bagnata 'wet flame' ([N Prt]) or infuocato gelo 'inflamed frost' ([Prt N]). We found mostly past participles (see the aforementioned examples), but also a few present participles (e.g. prossimità distanziante 'distancing proximity').

The third most frequent structure in terms of number of oxymorons is Sentence (S). The examples belonging to this class emerged especially (though not entirely) through the exploration of the Word Sketch function (cf. Section 2). The antonymous nouns in our pairs were found both in copular sentences (e.g. l'amore è odio 'love is hate', il silenzio è rumore 'the silence is noise') and in subject-verb sentences (e.g. il silenzio grida 'the silence screams', il buio illumina 'the dark illuminates (something)'). Some sentence-level oxymorons are borderline cases, since they could be argued to qualify as paradoxes rather than oxymorons (e.g. il buio è luce "the dark is the light'). We decided to keep them, since the divide between oxymorons and paradoxes is not so clear(-cut): as Flayih (2009) claims, the "oxymoron is sometimes taken as "condensed paradox' and paradox as 'expanded oxymoron"”.

We also retrieved a considerable number of adverbial oxymorons of the [Adv A] type (e.g. allegramente depresso 'cheerfully depressed', luminosamente oscuro 'brightly dark') - which is relevant also in terms of the number of antonymous pairs it represents (16 out of 17, cf. Table 2) - and some [N Prep N] oxymoronic expressions, such as la tenebra della luce 'darkness of the light'. As for the latter category, all examples contain the 
preposition $d i$ ' 'of', except for il silenzio nel rumore 'the silence into the noise', which contains in 'in'.

Finally, we found other less common structures, such as [A A] (e.g. fredda calda 'cold hot'), [V V] (where the second verb is a gerund, e.g. gridare tacendo 'to shout being silent'), [V A] (e.g. urlare muto 'to scream (staying) mute'), and [V Prep N] (e.g. urlare in silenzio 'to scream in silence').

\subsection{Antonymous pairs}

As for the antonymous pairs taken into consideration (Table 1), we observe a rather unequal distribution in our data in terms of their ability to create oxymorons. Some pairs - such as caldo $\sim$ freddo $($ hot $\sim$ cold), silenzio $\sim$ rumore (silence $\sim$ noise) or felicità $\sim$ infelicità (happiness $\sim$ unhappiness) - generate a high number of oxymoronic constructions, whereas others are definitely less exploited, like coraggio $\sim$ paura (bravery $\sim$ fear), guerra $\sim$ pace (war $\sim$ peace) or leggerezza $\sim$ pesantezza (lightness $\sim$ heaviness). Overall, the pairs with the higher number of oxymorons are also those displaying a wider array of syntactic structures (Table 2), but the differences are not so great. All the pairs are represented by at least 3 structures (out of 9 possibilities).

The complete quantitative picture is given in Table 3, where antonymous pairs are reported in English translation (like in the Appendix) for convenience.

\begin{tabular}{lrr}
\hline Antonymous pair & $\begin{array}{r}\text { N. of } \\
\text { oxymorons }\end{array}$ & $\begin{array}{r}\text { N. of } \\
\text { syntactic } \\
\text { structures }\end{array}$ \\
\hline hot-cold & 87 & 5 \\
silence-noise & 85 & 8 \\
happiness-unhappiness & 50 & 4 \\
light-dark & 30 & 5 \\
distance-proximity & 19 & 4 \\
love-hate & 18 & 5 \\
life-death & 13 & 4 \\
reality-unreality & 11 & 5 \\
wealth-poverty & 10 & 5 \\
sweetness-bitterness & 10 & 3 \\
slowness-speed & 9 & 3 \\
bravery-fear & 8 & 4 \\
activity-passivity & 7 & 3 \\
justice-injustice & 6 & 4 \\
war-peace & 5 & 4 \\
emptiness-fullness & 5 & 3 \\
lightness-heaviness & 3 & 3 \\
\hline Total & $\mathbf{3 7 6}$ & $\mathbf{9}$ \\
\hline
\end{tabular}

Table 3: Number of oxymorons and syntactic structures per each antonymous pair.
At first glance there does not seem to be a strong and clear driving principle behind the unequal distribution of the pairs in terms of semantics. For instance, we find abstract concepts (e.g. happiness $\sim$ unhappiness, justice $\sim$ injustice) or sensorial concepts (e.g. hot $\sim$ cold, lightness $\sim$ heaviness) at various points of the list in Table 3. However, a more fine-grained semantic analysis and a larger dataset would be necessary to draw more solid conclusions.

Of course, the higher number of semantically related lexemes investigated for some pairs plays a clear role (cf. Section 2). Also the entrenchment of some notorious cases might be relevant. Take for instance silenzio assordante 'deafening silence', which occurs 2564 times in the itTenTen 16 corpus (plus 1517 times in the reverse adjective-noun order: assordante silenzio). The high token frequency of this specific oxymoron may favor the creation of new oxymorons in the very same conceptual domain (silence $\sim$ noise).

\subsection{Morphosyntactic variability}

Although the morphosyntactic variation of oxymorons is not the focus of the present study, we can preliminary observe that, according to the data we collected so far, oxymorons are rather flexible structures. In other words, contrary to many multiword expressions (cf., among many others, Sag et al. 2002), oxymorons seem to show a low degree of fixedness.

Many combinations of a noun and an adjective are attested in both orders, although with different frequency (remember that noun-adjective is more neutral than adjective-noun): see the couple silenzio assordante vs. assordante silenzio mentioned at the end of Section 3.2, or tenebra luminosa (9 tokens in itTenTen16) vs. luminosa tenebra (4 tokens in itTenTen16) 'bright shadow'.

In [N Prep $\mathrm{N}$ ] oxymorons, the second (nonhead) noun may occur in both singular and plural (contrary to Italian multiword expressions belonging to the same pattern, where the non-head noun is morphologically fixed, cf. Masini 2009), although the singular form is generally preferred, e.g.: suono del silenzio 'sound of the silence' ( 380 tokens in itTenTen16) vs. suono dei silenzi 'sound of the silences' (7 tokens in itTenTen16).

As for sentential oxymorons, we often found different configurations for the same pair of items; for instance, silenzio 'silence' and rumore 'noise' are found as: il silenzio è un rumore (che...) 
'silence is a noise (that...)', il silenzio è rumore 'silence is noise', il silenzio è il rumore (di...) 'silence is the noise (of...)' (all these variants are reported as a single entry - il silenzio è rumore 'silence is noise' - in the Appendix).

\section{A complementary method}

Another complementary technique to harvest oxymorons from corpora is, quite trivially, to search the word ossimoro 'oxymoron'. We noticed that, when they come across or use (what they believe to be) an oxymoron, speakers tend to comment on it metalinguistically, as in: una guerra santa (grandissimo ossimoro) 'a holy war (huge oxymoron)' (from CORIS). This behavior allows to detect oxymorons which would probably be missed otherwise.

To test this method we searched the string [. *ossimor.*] in CORIS (retrieving 223 hits). Upon preliminary manual checking, we found a number of valid oxymorons. A few were already included in our list, like tenebra luminosissima 'very bright shadow' (cf. tenebra luminosa 'bright shadow' in the Appendix). Another few were examples, related to one of the antonymous pairs we considered (cf. Table 1), which were not retrieved by our method, like boato di silenzio 'roar of silence' (belonging to the silenzio rumore 'silence $\sim$ noise' pair).

Most cases, however, were completely new oxymorons. Some could have been ideally identified with our method based on antonymous pairs (e.g. normalmente eccezionali 'normally exceptional', piangendo rido 'I laugh crying', caoticamente ordinate 'chaotically tidy'), but many others turned out to be hardly foreseeable and heavily dependent on context and on the speaker's beliefs and communicative intentions. Take for instance: A Cala del Faro, villaggio superchic di Porto Cervo, l'ultimo business è un ossimoro applicato al calcio: il ritiro mondano 'In Cala del Faro, a superchic village at Porto Cervo, the last business is an oxymoron applied to football: the social training camp', where the writer emphasizes the contradiction in hosting a ritiro 'training camp' (lit. retirement) in such a voguish and social place like Porto Cervo. Or: vi attendono minacciosi decine di ristoranti tibetani, praticamente un ossimoro [...] non essendo propriamente gli altopiani tibetani un paradiso dell'enogastronomia '[there] tens of threatening Tibetan restaurants are waiting for you, basically an oxymoron $[\ldots]$ since Tibetan uplands are not exactly the paradise of food and wine', where the writer's bad opinion about Tibetan food and wine is ironically expressed.

Therefore, this method might be especially promising for identifying creative, highly contextual oxymorons. In this respect, social media platforms are definitely one of the sources to be used for this kind of research, which we leave for future studies.

\section{Future challenges}

This short paper illustrates the results of an investigation aimed at tracking down oxymoronic constructions in corpora of written Italian. Far from being exhaustive, this is just a preliminary attempt, resulting in an initial list of 376 oxymorons that needs to be enriched.

On the one hand, this enrichment may be pursued by adding more antonymous pairs under the current approach, which however heavily relies on manual work at various stages and would benefit from a higher degree of automation.

On the other hand, different methods should be devised and tested for the (we suspect) wide set of oxymorons that cannot be traced back to antonymous pairs. For instance, some oxymorons actually contain synonymous or even identical elements, within specific structures, such as suoni senza suono 'sounds without sound' (retrieved from itTenTen16). More interestingly, as Gibbs (1993: 269) observes, "oxymora are frequently found in everyday speech, many of them barely noticed as such, for example, 'intense apathy,' 'internal exile,' 'man child,' 'loyal opposition,' 'plastic glasses,' 'guest host,' and so on. The ubiquity of these figures suggests some underlying ability to conceive of ideas, objects, and events in oxymoronic terms." But - we add - it also suggests that the detection of these tropes is yet another challenge for automatic extraction, especially considering that some are heavily contextual.

Moreover, many oxymorons are "jocular", as Horn (2018) observes, mentioning cases such as military intelligence, congressional ethics, airplane food and open secrets. Given this, a deeper investigation of oxymorons looks even more desirable. Beside advancing our knowledge of figurative language in general, it would have clear benefits for other mainstream challenges in NLP, like the detection of humor, irony and sarcasm. 


\section{References}

Walter Charles and George Miller. 1989. Contexts of antonymous adjectives. Applied Psycholinguistics, 10(3):357-375.

Reja'a M. Flayih. 2009. A linguistic study of oxymoron. Journal of Kerbala University, 7(3):3040.

Raymond W. Gibbs, Jr. 1993. Process and products in making sense of tropes. In Metaphor and Thought $\left(2^{\text {nd }}\right.$ ed.), Andrew Ortony (ed.). Cambridge: Cambridge University Press, pages 252-276.

Raymond W. Gibbs, Jr. and Lydia R. Kearney. 1994. When parting is such sweet sorrow: The comprehension and appreciation of oxymora. Journal of Psycholinguistic Research, 23(1):75-89.

Laurence R. Horn. 2018. Contradiction. In The Stanford Encyclopedia of Philosophy (Winter 2018 Edition), Edward N. Zalta (ed.).

Steven Jones. 2002. Antonymy: A Corpus-based Perspective. London and New York: Routledge.

John S. Justeson and Slava M. Katz. 1991. Cooccurrences of antonymous adjectives and their contexts. Computational Linguistics, 17(1):1-19.

Nataša Kostić. 2017. Adjectival antonyms in discourse: A corpus study of scalar and complementary antonyms. Folia Linguistica, 51(3):587-610.

Anna Lobanova. 2012. The Anatomy of Antonymy: A Corpus-driven Approach. Groningen University $\mathrm{PhD}$ dissertation.

Francesca Masini. 2009. Phrasal lexemes, compounds and phrases: A constructionist perspective. Word Structure, 2(2):254-271.

Lynne M. Murphy. 2006. Antonyms as lexical constructions: Or, why paradigmatic construction is not an oxymoron. Constructions, SV1-8/2006.

Francesca Ramaglia. 2010. Aggettivi. In Enciclopedia dell'Italiano. Treccani.

Rema Rossini Favretti, Fabio Tamburini and Cristiana De Santis. 2002. CORIS/CODIS: A corpus of written Italian based on a defined and a dynamic model. In $A$ Rainbow of Corpora: Corpus Linguistics and the Languages of the World, Andrew Wilson, P. Rayson and A. M. McEnery (eds.). Munich: Lincom-Europa, pages 27-38.

Ivan A. Sag, Timothy Baldwin, Francis Bond, Ann Copestake and Dan Flickinger .2002. Multiword expressions: A pain in the neck for NLP. In Computational linguistics and intelligent text processing: Third International Conference: CICLing-2002 (LNCS 2276), Alexander Gelbukh (ed.). Berlin/Heidelberg: Springer, pages 1-15.

Yeshayahu Shen. 1987. On the structure and understanding of poetic oxymoron. Poetics Today, 8(1):105-122.

Raffaele Simone (ed.). 2010. Grande Dizionario Analogico della Lingua Italiana (CD-ROM). Torino: UTET.

\section{A Appendix: Italian oxymorons 1.0}

This Appendix contains the lemmatized version of the 376 oxymorons we retrieved, ordered by "Antonymous pair" (the pairs appear in their English translation). As for sentential oxymorons displaying variants (cf. Section 3.3), we chose to report a single version as representative of all variants, for the sake of simplicity.

\begin{tabular}{|c|c|c|c|c|}
\hline $\begin{array}{l}\text { Italian } \\
\text { oxymoron }\end{array}$ & $\begin{array}{l}\text { English } \\
\text { translation }\end{array}$ & Structure & $\begin{array}{l}\text { Antonymous } \\
\text { pair }\end{array}$ & Corpus \\
\hline $\begin{array}{l}\text { attiva } \\
\text { passività }\end{array}$ & active passivity & $\mathrm{AN}$ & $\begin{array}{l}\text { activity- } \\
\text { passivity }\end{array}$ & itTenTen 16 \\
\hline $\begin{array}{l}\text { attivamente } \\
\text { passivo }\end{array}$ & actively passive & Adv A & $\begin{array}{l}\text { activity- } \\
\text { passivity }\end{array}$ & itTenTen16 \\
\hline $\begin{array}{l}\text { attività } \\
\text { inattiva }\end{array}$ & inactive activity & $\mathrm{NA}$ & $\begin{array}{l}\text { activity- } \\
\text { passivity }\end{array}$ & itTenTen 16 \\
\hline $\begin{array}{l}\text { attività } \\
\text { passiva }\end{array}$ & passive activity & $\mathrm{NA}$ & $\begin{array}{l}\text { activity- } \\
\text { passivity }\end{array}$ & $\begin{array}{l}\text { CORIS + } \\
\text { itTenTen16 }\end{array}$ \\
\hline $\begin{array}{l}\text { passiva } \\
\text { attività }\end{array}$ & passive activity & A N & $\begin{array}{l}\text { activity- } \\
\text { passivity }\end{array}$ & itTenTen16 \\
\hline $\begin{array}{l}\text { passivamente } \\
\text { attivo }\end{array}$ & passively active & Adv A & $\begin{array}{l}\text { activity- } \\
\text { passivity }\end{array}$ & itTenTen 16 \\
\hline $\begin{array}{l}\text { passività } \\
\text { attiva }\end{array}$ & active passivity & $\mathrm{NA}$ & $\begin{array}{l}\text { activity- } \\
\text { passivity }\end{array}$ & $\begin{array}{l}\text { CORIS + } \\
\text { itTenTen16 }\end{array}$ \\
\hline $\begin{array}{l}\text { coraggiosa } \\
\text { paura }\end{array}$ & brave fear & A N & bravery-fear & itTenTen 16 \\
\hline $\begin{array}{l}\text { coraggiosame } \\
\text { nte pauroso }\end{array}$ & bravely scared & Adv A & bravery-fear & itTenTen16 \\
\hline $\begin{array}{l}\text { coraggiosame } \\
\text { nte vigliacco }\end{array}$ & bravely coward & Adv A & bravery-fear & itTenTen 16 \\
\hline $\begin{array}{l}\text { coraggiosame } \\
\text { nte vile }\end{array}$ & $\begin{array}{l}\text { bravely } \\
\text { cowardly }\end{array}$ & Adv A & bravery-fear & itTenTen 16 \\
\hline $\begin{array}{l}\text { il coraggio } \\
\text { della paura }\end{array}$ & $\begin{array}{l}\text { the bravery of } \\
\text { fear }\end{array}$ & N Prep N & bravery-fear & itTenTen 16 \\
\hline $\begin{array}{l}\text { paura } \\
\text { impavida }\end{array}$ & fearless fear & $\mathrm{NA}$ & bravery-fear & CORIS \\
\hline $\begin{array}{l}\text { pauroso } \\
\text { coraggio }\end{array}$ & fearful bravery & $\mathrm{AN}$ & bravery-fear & CORIS \\
\hline vile coraggio & $\begin{array}{l}\text { cowardly } \\
\text { bravery }\end{array}$ & $\mathrm{AN}$ & bravery-fear & itTenTen 16 \\
\hline $\begin{array}{l}\text { distante } \\
\text { prossimità }\end{array}$ & far proximity & A N & $\begin{array}{l}\text { distance- } \\
\text { proximity }\end{array}$ & itTenTen 16 \\
\hline $\begin{array}{l}\text { distante } \\
\text { vicinanza }\end{array}$ & far closeness & A N & $\begin{array}{l}\text { distance- } \\
\text { proximity }\end{array}$ & itTenTen16 \\
\hline $\begin{array}{l}\text { distanza } \\
\text { vicina }\end{array}$ & near distance & $\mathrm{NA}$ & $\begin{array}{l}\text { distance- } \\
\text { proximity }\end{array}$ & itTenTen 16 \\
\hline $\begin{array}{l}\text { la distanza } \\
\text { avvicina }\end{array}$ & $\begin{array}{l}\text { distance brings } \\
\text { (sth) closer }\end{array}$ & $\mathrm{S}$ & $\begin{array}{l}\text { distance- } \\
\text { proximity }\end{array}$ & itTenTen 16 \\
\hline $\begin{array}{l}\text { la lontananza } \\
\text { avvicina }\end{array}$ & $\begin{array}{l}\text { distance brings } \\
\text { (sth) closer }\end{array}$ & $\mathrm{S}$ & $\begin{array}{l}\text { distance- } \\
\text { proximity }\end{array}$ & itTenTen16 \\
\hline $\begin{array}{l}\text { la vicinanza } \\
\text { allontana }\end{array}$ & $\begin{array}{l}\text { proximity pulls } \\
\text { (sth) away }\end{array}$ & $\mathrm{S}$ & $\begin{array}{l}\text { distance- } \\
\text { proximity }\end{array}$ & itTenTen 16 \\
\hline $\begin{array}{l}\text { lontana } \\
\text { vicinanza }\end{array}$ & $\begin{array}{l}\text { distant } \\
\text { proximity }\end{array}$ & A N & $\begin{array}{l}\text { distance- } \\
\text { proximity }\end{array}$ & itTenTen 16 \\
\hline $\begin{array}{l}\text { lontanamente } \\
\text { vicino }\end{array}$ & remotely close & Adv A & $\begin{array}{l}\text { distance- } \\
\text { proximity }\end{array}$ & itTenTen 16 \\
\hline $\begin{array}{l}\text { lontananza } \\
\text { vicina }\end{array}$ & near distance & N A & $\begin{array}{l}\text { distance- } \\
\text { proximity }\end{array}$ & itTenTen 16 \\
\hline $\begin{array}{l}\text { prossimità } \\
\text { distante }\end{array}$ & far proximity & $\mathrm{NA}$ & $\begin{array}{l}\text { distance- } \\
\text { proximity }\end{array}$ & itTenTen16 \\
\hline $\begin{array}{l}\text { prossimità } \\
\text { distanziante }\end{array}$ & $\begin{array}{l}\text { distancing } \\
\text { proximity }\end{array}$ & $\mathrm{NA}$ & $\begin{array}{l}\text { distance- } \\
\text { proximity }\end{array}$ & itTenTen 16 \\
\hline $\begin{array}{l}\text { prossimità } \\
\text { lontana }\end{array}$ & far proximity & $\mathrm{NA}$ & $\begin{array}{l}\text { distance- } \\
\text { proximity }\end{array}$ & itTenTen 16 \\
\hline $\begin{array}{l}\text { prossimità } \\
\text { remota }\end{array}$ & $\begin{array}{l}\text { distant } \\
\text { proximity }\end{array}$ & NA & $\begin{array}{l}\text { distance- } \\
\text { proximity }\end{array}$ & itTenTen16 \\
\hline $\begin{array}{l}\text { remota } \\
\text { prossimità }\end{array}$ & $\begin{array}{l}\text { distant } \\
\text { proximity }\end{array}$ & A N & $\begin{array}{l}\text { distance- } \\
\text { proximity }\end{array}$ & itTenTen 16 \\
\hline
\end{tabular}




\begin{tabular}{|c|c|c|c|c|}
\hline $\begin{array}{l}\text { vicina } \\
\text { distanza } \\
\end{array}$ & near distance & $\mathrm{AN}$ & $\begin{array}{l}\text { distance- } \\
\text { proximity }\end{array}$ & $\begin{array}{l}\text { CORIS + } \\
\text { itTenTen16 }\end{array}$ \\
\hline $\begin{array}{l}\text { vicina } \\
\text { lontananza }\end{array}$ & near distance & A N & $\begin{array}{l}\text { distance- } \\
\text { proximity }\end{array}$ & itTenTen 16 \\
\hline $\begin{array}{l}\text { vicinanza } \\
\text { distante }\end{array}$ & far proximity & $\mathrm{NA}$ & $\begin{array}{l}\text { distance- } \\
\text { proximity }\end{array}$ & itTenTen 16 \\
\hline $\begin{array}{l}\text { vicinanza } \\
\text { lontana }\end{array}$ & $\begin{array}{l}\text { distant } \\
\text { proximity }\end{array}$ & $\mathrm{NA}$ & $\begin{array}{l}\text { distance- } \\
\text { proximity }\end{array}$ & itTenTen 16 \\
\hline $\begin{array}{l}\text { vicinanza } \\
\text { remota }\end{array}$ & $\begin{array}{l}\text { remote } \\
\text { proximity }\end{array}$ & N A & $\begin{array}{l}\text { distance- } \\
\text { proximity }\end{array}$ & CORIS \\
\hline $\begin{array}{l}\text { il vuoto } \\
\text { riempie }\end{array}$ & $\begin{array}{l}\text { the void fills } \\
\text { (sth) up }\end{array}$ & $\mathrm{S}$ & $\begin{array}{l}\text { emptiness- } \\
\text { fullness }\end{array}$ & $\begin{array}{l}\text { CORIS + } \\
\text { itTenTen16 }\end{array}$ \\
\hline $\begin{array}{l}\text { pienamente } \\
\text { svuotato }\end{array}$ & fully emptied & Adv A & $\begin{array}{l}\text { emptiness- } \\
\text { fullness }\end{array}$ & itTenTen 16 \\
\hline pieno vuoto & full void & $\mathrm{AN}$ & $\begin{array}{l}\text { emptiness- } \\
\text { fullness }\end{array}$ & itTenTen 16 \\
\hline vuoto colmo & full void & $\mathrm{NA}$ & $\begin{array}{l}\text { emptiness- } \\
\text { fullness }\end{array}$ & itTenTen 16 \\
\hline vuoto pieno & full void & $\mathrm{NA}$ & $\begin{array}{l}\text { emptiness- } \\
\text { fullness }\end{array}$ & $\begin{array}{l}\text { CORIS + } \\
\text { itTenTen16 }\end{array}$ \\
\hline $\begin{array}{l}\text { addolorata } \\
\text { contentezza }\end{array}$ & $\begin{array}{l}\text { sorrowful } \\
\text { gladness }\end{array}$ & A N & $\begin{array}{l}\text { happiness- } \\
\text { unhappiness }\end{array}$ & itTenTen 16 \\
\hline $\begin{array}{l}\text { allegra } \\
\text { depressione }\end{array}$ & $\begin{array}{l}\text { merry } \\
\text { depression }\end{array}$ & $\mathrm{AN}$ & $\begin{array}{l}\text { happiness- } \\
\text { unhappiness }\end{array}$ & itTenTen 16 \\
\hline $\begin{array}{l}\text { allegra } \\
\text { disperazione }\end{array}$ & merry despair & $\mathrm{AN}$ & $\begin{array}{l}\text { happiness- } \\
\text { unhappiness }\end{array}$ & itTenTen 16 \\
\hline $\begin{array}{l}\text { allegra } \\
\text { infelicità }\end{array}$ & $\begin{array}{l}\text { merry } \\
\text { unhappiness }\end{array}$ & $\mathrm{AN}$ & $\begin{array}{l}\text { happiness- } \\
\text { unhappiness }\end{array}$ & itTenTen 16 \\
\hline $\begin{array}{l}\text { allegra } \\
\text { tristezza }\end{array}$ & merry sadness & $\mathrm{AN}$ & $\begin{array}{l}\text { happiness- } \\
\text { unhappiness }\end{array}$ & itTenTen 16 \\
\hline $\begin{array}{l}\text { allegramente } \\
\text { depresso }\end{array}$ & $\begin{array}{l}\text { cheerfully } \\
\text { depressed }\end{array}$ & Adv A & $\begin{array}{l}\text { happiness- } \\
\text { unhappiness }\end{array}$ & itTenTen 16 \\
\hline $\begin{array}{l}\text { allegramente } \\
\text { disperato }\end{array}$ & $\begin{array}{l}\text { cheerfully } \\
\text { desperate }\end{array}$ & Adv A & $\begin{array}{l}\text { happiness- } \\
\text { unhappiness }\end{array}$ & itTenTen 16 \\
\hline $\begin{array}{l}\text { allegramente } \\
\text { triste }\end{array}$ & cheerfully sad & Adv A & $\begin{array}{l}\text { happiness- } \\
\text { unhappiness }\end{array}$ & itTenTen 16 \\
\hline $\begin{array}{l}\text { allegria } \\
\text { disperata }\end{array}$ & desperate glee & $\mathrm{NA}$ & $\begin{array}{l}\text { happiness- } \\
\text { unhappiness }\end{array}$ & $\begin{array}{l}\text { CORIS + } \\
\text { itTenTen16 }\end{array}$ \\
\hline $\begin{array}{l}\text { allegria } \\
\text { scontenta }\end{array}$ & displeased glee & $\mathrm{NA}$ & $\begin{array}{l}\text { happiness- } \\
\text { unhappiness }\end{array}$ & itTenTen 16 \\
\hline allegria triste & sad glee & $\mathrm{NA}$ & $\begin{array}{l}\text { happiness- } \\
\text { unhappiness }\end{array}$ & itTenTen 16 \\
\hline cupa felicità & $\begin{array}{l}\text { gloomy } \\
\text { happiness }\end{array}$ & $\mathrm{AN}$ & $\begin{array}{l}\text { happiness- } \\
\text { unhappiness }\end{array}$ & $\begin{array}{l}\text { CORIS + } \\
\text { itTenTen16 }\end{array}$ \\
\hline $\begin{array}{l}\text { disperata } \\
\text { allegria }\end{array}$ & desperate glee & $\mathrm{AN}$ & $\begin{array}{l}\text { happiness- } \\
\text { unhappiness }\end{array}$ & $\begin{array}{l}\text { CORIS + } \\
\text { itTenTen16 }\end{array}$ \\
\hline $\begin{array}{l}\text { disperata } \\
\text { felicità }\end{array}$ & $\begin{array}{l}\text { desperate } \\
\text { happiness }\end{array}$ & $\mathrm{AN}$ & $\begin{array}{l}\text { happiness- } \\
\text { unhappiness }\end{array}$ & itTenTen 16 \\
\hline disperata gioia & desperate joy & $\mathrm{AN}$ & $\begin{array}{l}\text { happiness- } \\
\text { unhappiness }\end{array}$ & itTenTen 16 \\
\hline $\begin{array}{l}\text { disperatament } \\
\text { e allegro }\end{array}$ & $\begin{array}{l}\text { desperately } \\
\text { merry }\end{array}$ & Adv A & $\begin{array}{l}\text { happiness- } \\
\text { unhappiness }\end{array}$ & itTenTen 16 \\
\hline $\begin{array}{l}\text { disperatament } \\
\text { e contento }\end{array}$ & $\begin{array}{l}\text { desperately } \\
\text { glad }\end{array}$ & Adv A & $\begin{array}{l}\text { happiness- } \\
\text { unhappiness }\end{array}$ & itTenTen 16 \\
\hline $\begin{array}{l}\text { disperatament } \\
\text { e felice }\end{array}$ & $\begin{array}{l}\text { desperately } \\
\text { happy }\end{array}$ & Adv A & $\begin{array}{l}\text { happiness- } \\
\text { unhappiness }\end{array}$ & $\begin{array}{l}\text { CORIS + } \\
\text { itTenTen16 }\end{array}$ \\
\hline $\begin{array}{l}\text { disperatament } \\
\text { e gioioso }\end{array}$ & $\begin{array}{l}\text { desperately } \\
\text { joyful }\end{array}$ & Adv A & $\begin{array}{l}\text { happiness- } \\
\text { unhappiness }\end{array}$ & itTenTen 16 \\
\hline $\begin{array}{l}\text { disperazione } \\
\text { allegra }\end{array}$ & merry despair & N A & $\begin{array}{l}\text { happiness- } \\
\text { unhappiness }\end{array}$ & itTenTen 16 \\
\hline dolore gioioso & joyful pain & $\mathrm{NA}$ & $\begin{array}{l}\text { happiness- } \\
\text { unhappiness }\end{array}$ & itTenTen 16 \\
\hline $\begin{array}{l}\text { felice } \\
\text { disperazione }\end{array}$ & happy despair & $\mathrm{AN}$ & $\begin{array}{l}\text { happiness- } \\
\text { unhappiness }\end{array}$ & itTenTen 16 \\
\hline $\begin{array}{l}\text { felice } \\
\text { infelicità }\end{array}$ & $\begin{array}{l}\text { happy } \\
\text { unhappiness }\end{array}$ & $\mathrm{AN}$ & $\begin{array}{l}\text { happiness- } \\
\text { unhappiness }\end{array}$ & itTenTen 16 \\
\hline felice tristezza & happy sadness & $\mathrm{AN}$ & $\begin{array}{l}\text { happiness- } \\
\text { unhappiness }\end{array}$ & itTenTen 16 \\
\hline $\begin{array}{l}\text { felicemente } \\
\text { infelice }\end{array}$ & $\begin{array}{l}\text { happily } \\
\text { unhappy }\end{array}$ & Adv A & $\begin{array}{l}\text { happiness- } \\
\text { unhappiness }\end{array}$ & itTenTen 16 \\
\hline $\begin{array}{l}\text { felicemente } \\
\text { triste }\end{array}$ & happily sad & Adv A & $\begin{array}{l}\text { happiness- } \\
\text { unhappiness }\end{array}$ & itTenTen 16 \\
\hline $\begin{array}{l}\text { felicità } \\
\text { disperata }\end{array}$ & $\begin{array}{l}\text { desperate } \\
\text { happiness }\end{array}$ & N A & $\begin{array}{l}\text { happiness- } \\
\text { unhappiness }\end{array}$ & itTenTen 16 \\
\hline felicità triste & sad happiness & N A & $\begin{array}{l}\text { happiness- } \\
\text { unhappiness }\end{array}$ & itTenTen 16 \\
\hline $\begin{array}{l}\text { gaia } \\
\text { disperazione }\end{array}$ & cheerful despair & $\mathrm{AN}$ & $\begin{array}{l}\text { happiness- } \\
\text { unhappiness }\end{array}$ & itTenTen 16 \\
\hline gaia tristezza & $\begin{array}{l}\text { cheerful } \\
\text { sadness }\end{array}$ & $\mathrm{AN}$ & $\begin{array}{l}\text { happiness- } \\
\text { unhappiness }\end{array}$ & itTenTen 16 \\
\hline $\begin{array}{l}\text { gaiamente } \\
\text { infelice }\end{array}$ & gaily unhappy & Adv A & $\begin{array}{l}\text { happiness- } \\
\text { unhappiness }\end{array}$ & itTenTen 16 \\
\hline gioia cupa & gloomy joy & N A & $\begin{array}{l}\text { happiness- } \\
\text { unhappiness }\end{array}$ & $\begin{array}{l}\text { CORIS + } \\
\text { itTenTen16 }\end{array}$ \\
\hline gioia disperata & desperate joy & N A & $\begin{array}{l}\text { happiness- } \\
\text { unhappiness }\end{array}$ & $\begin{array}{l}\text { CORIS + } \\
\text { itTenTen16 }\end{array}$ \\
\hline
\end{tabular}

\begin{tabular}{|c|c|c|c|c|}
\hline $\begin{array}{l}\text { gioia } \\
\text { sconsolata }\end{array}$ & sorrowful joy & $\mathrm{NA}$ & $\begin{array}{l}\text { happiness- } \\
\text { unhappiness }\end{array}$ & itTenTen 16 \\
\hline gioia triste & sad joy & $\mathrm{NA}$ & $\begin{array}{l}\text { happiness- } \\
\text { unhappiness }\end{array}$ & $\begin{array}{l}\text { CORIS + } \\
\text { itTenTen16 }\end{array}$ \\
\hline $\begin{array}{l}\text { gioiosa } \\
\text { disperazione }\end{array}$ & joyful despair & A N & $\begin{array}{l}\text { happiness- } \\
\text { unhappiness }\end{array}$ & itTenTen16 \\
\hline $\begin{array}{l}\text { gioiosa } \\
\text { tristezza }\end{array}$ & joyful sadness & $\mathrm{AN}$ & $\begin{array}{l}\text { happiness- } \\
\text { unhappiness }\end{array}$ & itTenTen 16 \\
\hline $\begin{array}{l}\text { gioiosamente } \\
\text { disperato }\end{array}$ & $\begin{array}{l}\text { joyously } \\
\text { desperate }\end{array}$ & Adv A & $\begin{array}{l}\text { happiness- } \\
\text { unhappiness }\end{array}$ & itTenTen 16 \\
\hline $\begin{array}{l}\text { infelice } \\
\text { felicità }\end{array}$ & $\begin{array}{l}\text { unhappy } \\
\text { happiness }\end{array}$ & $\mathrm{AN}$ & $\begin{array}{l}\text { happiness- } \\
\text { unhappiness }\end{array}$ & $\begin{array}{l}\text { CORIS + } \\
\text { itTenTen16 }\end{array}$ \\
\hline $\begin{array}{l}\text { l'allegria (lo) } \\
\text { fa triste }\end{array}$ & $\begin{array}{l}\text { cheerfulness } \\
\text { makes (sb) sad }\end{array}$ & $\mathrm{S}$ & $\begin{array}{l}\text { happiness- } \\
\text { unhappiness }\end{array}$ & itTenTen 16 \\
\hline $\begin{array}{l}\text { la gioia è } \\
\text { dolore }\end{array}$ & joy is pain & $\mathrm{S}$ & $\begin{array}{l}\text { happiness- } \\
\text { unhappiness }\end{array}$ & itTenTen 16 \\
\hline $\begin{array}{l}\text { la tristezza } \\
\text { (lo) fa allegro }\end{array}$ & $\begin{array}{l}\text { sadness makes } \\
\text { (sb) merry }\end{array}$ & $\mathrm{S}$ & $\begin{array}{l}\text { happiness- } \\
\text { unhappiness }\end{array}$ & itTenTen 16 \\
\hline $\begin{array}{l}\text { sconsolata } \\
\text { allegria }\end{array}$ & $\begin{array}{l}\text { sorrowful } \\
\text { cheerfulness }\end{array}$ & $\mathrm{AN}$ & $\begin{array}{l}\text { happiness- } \\
\text { unhappiness }\end{array}$ & itTenTen 16 \\
\hline triste allegria & $\begin{array}{l}\text { sad } \\
\text { cheerfulness }\end{array}$ & A N & $\begin{array}{l}\text { happiness- } \\
\text { unhappiness }\end{array}$ & itTenTen 16 \\
\hline $\begin{array}{l}\text { triste } \\
\text { contentezza }\end{array}$ & sad gladness & $\mathrm{AN}$ & $\begin{array}{l}\text { happiness- } \\
\text { unhappiness }\end{array}$ & itTenTen 16 \\
\hline triste felicità & sad happiness & A N & $\begin{array}{l}\text { happiness- } \\
\text { unhappiness }\end{array}$ & itTenTen 16 \\
\hline triste gaiezza & sad gaiety & $\mathrm{AN}$ & $\begin{array}{l}\text { happiness- } \\
\text { unhappiness }\end{array}$ & itTenTen 16 \\
\hline triste gioia & sad joy & $\mathrm{AN}$ & $\begin{array}{l}\text { happiness- } \\
\text { unhappiness }\end{array}$ & itTenTen 16 \\
\hline $\begin{array}{l}\text { tristezza } \\
\text { allegra }\end{array}$ & merry sadness & $\mathrm{NA}$ & $\begin{array}{l}\text { happiness- } \\
\text { unhappiness }\end{array}$ & itTenTen 16 \\
\hline tristezza felice & happy sadness & $\mathrm{NA}$ & $\begin{array}{l}\text { happiness- } \\
\text { unhappiness }\end{array}$ & $\begin{array}{l}\text { CORIS + } \\
\text { itTenTen16 }\end{array}$ \\
\hline acqua ignea & igneous water & $\mathrm{NA}$ & hot-cold & itTenTen16 \\
\hline acquoso fuoco & watery fire & A N & hot-cold & itTenTen 16 \\
\hline $\begin{array}{l}\text { ardente } \\
\text { ghiaccio }\end{array}$ & burning ice & $\mathrm{AN}$ & hot-cold & itTenTen16 \\
\hline $\begin{array}{l}\text { ardente } \\
\text { pioggia }\end{array}$ & blazing rain & $\mathrm{AN}$ & hot-cold & itTenTen 16 \\
\hline $\begin{array}{l}\text { calda } \\
\text { freddezza }\end{array}$ & warm coldness & $\mathrm{AN}$ & hot-cold & itTenTen 16 \\
\hline calda fresca & hot cold & A A & hot-cold & itTenTen 16 \\
\hline calda neve & hot snow & A N & hot-cold & itTenTen 16 \\
\hline caldo freddo & hot cold & $\mathrm{AN}$ & hot-cold & $\begin{array}{l}\text { CORIS + } \\
\text { itTenTen16 }\end{array}$ \\
\hline caldo fresco & cool hot & $\mathrm{NA}$ & hot-cold & itTenTen16 \\
\hline caldo gelido & gelid hot & $\mathrm{NA}$ & hot-cold & itTenTen 16 \\
\hline caldo gelo & hot frost & A N & hot-cold & itTenTen 16 \\
\hline caldo ghiaccio & hot ice & $\mathrm{AN}$ & hot-cold & itTenTen 16 \\
\hline calore freddo & cold warmth & $\mathrm{NA}$ & hot-cold & $\begin{array}{l}\text { CORIS + } \\
\text { itTenTen16 }\end{array}$ \\
\hline calore gelido & icy warmth & N A & hot-cold & itTenTen 16 \\
\hline $\begin{array}{l}\text { calore } \\
\text { ghiacciato }\end{array}$ & frozen warmth & $\mathrm{NA}$ & hot-cold & itTenTen 16 \\
\hline $\begin{array}{l}\text { calorosamente } \\
\text { freddo }\end{array}$ & warmly cold & Adv A & hot-cold & itTenTen 16 \\
\hline $\begin{array}{l}\text { cocente } \\
\text { freddo }\end{array}$ & searing cold & AN & hot-cold & itTenTen16 \\
\hline $\begin{array}{l}\text { fiamma } \\
\text { bagnata }\end{array}$ & wet flame & $\mathrm{NA}$ & hot-cold & itTenTen16 \\
\hline $\begin{array}{l}\text { fiamma } \\
\text { congelata }\end{array}$ & frozen flame & $\mathrm{NA}$ & hot-cold & itTenTen 16 \\
\hline fiamma fredda & cold flame & $\mathrm{NA}$ & hot-cold & $\begin{array}{l}\text { CORIS + } \\
\text { itTenTen16 }\end{array}$ \\
\hline fiamma fresca & cool flame & $\mathrm{NA}$ & hot-cold & $\begin{array}{l}\text { CORIS + } \\
\text { itTenTen16 }\end{array}$ \\
\hline fiamma gelida & freezing flame & N A & hot-cold & itTenTen16 \\
\hline $\begin{array}{l}\text { fiamma } \\
\text { glaciale }\end{array}$ & glacial flame & $\mathrm{NA}$ & hot-cold & itTenTen16 \\
\hline fredda calda & cold hot & A A & hot-cold & itTenTen 16 \\
\hline fredda fiamma & cold flame & A N & hot-cold & $\begin{array}{l}\text { CORIS + } \\
\text { itTenTen16 }\end{array}$ \\
\hline $\begin{array}{l}\text { freddezza } \\
\text { calda }\end{array}$ & warm coldness & $\mathrm{NA}$ & hot-cold & itTenTen 16 \\
\hline $\begin{array}{l}\text { freddo } \\
\text { bollente }\end{array}$ & scalding cold & NA & hot-cold & itTenTen 16 \\
\hline freddo calore & cold warmth & $\mathrm{AN}$ & hot-cold & itTenTen 16 \\
\hline $\begin{array}{l}\text { freddo } \\
\text { caloroso }\end{array}$ & warm cold & NA & hot-cold & itTenTen 16 \\
\hline freddo fuoco & cold fire & A N & hot-cold & $\begin{array}{l}\text { CORIS + } \\
\text { itTenTen16 }\end{array}$ \\
\hline $\begin{array}{l}\text { freddo } \\
\text { incandescente }\end{array}$ & searing cold & $\mathrm{NA}$ & hot-cold & itTenTen 16 \\
\hline freddo torrido & scorching cold & N A & hot-cold & itTenTen 16 \\
\hline fresca calura & cool heat & $\mathrm{AN}$ & hot-cold & itTenTen 16 \\
\hline fresca fiamma & cool flame & $\mathrm{AN}$ & hot-cold & itTenTen 16 \\
\hline
\end{tabular}




\begin{tabular}{|c|c|c|c|c|}
\hline fresco caldo & cool hot & A N & hot-cold & itTenTen 16 \\
\hline fresco calore & cool warmth & AN & hot-cold & itTenTen16 \\
\hline fresco fuoco & cool fire & $\mathrm{AN}$ & hot-cold & itTenTen 16 \\
\hline fuoco acquoso & watery fire & $\mathrm{NA}$ & hot-cold & $\begin{array}{l}\text { CORIS + } \\
\text { itTenTen16 }\end{array}$ \\
\hline fuoco algido & cold fire & $\mathrm{NA}$ & hot-cold & $\begin{array}{l}\text { CORIS + } \\
\text { itTenTen16 }\end{array}$ \\
\hline $\begin{array}{l}\text { fuoco } \\
\text { congelato }\end{array}$ & frozen fire & N A & hot-cold & itTenTen 16 \\
\hline fuoco freddo & cold fire & $\mathrm{NA}$ & hot-cold & $\begin{array}{l}\text { CORIS + } \\
\text { itTenTen16 }\end{array}$ \\
\hline fuoco fresco & cool fire & $\mathrm{N} A$ & hot-cold & itTenTen 16 \\
\hline fuoco gelato & frozen fire & N A & hot-cold & itTenTen 16 \\
\hline fuoco gelido & icy fire & $\mathrm{NA}$ & hot-cold & $\begin{array}{l}\text { CORIS + } \\
\text { itTenTen16 }\end{array}$ \\
\hline $\begin{array}{l}\text { fuoco } \\
\text { ghiacciato }\end{array}$ & icy fire & $\mathrm{NA}$ & hot-cold & itTenTen16 \\
\hline gelida fiamma & freezing flame & A N & hot-cold & itTenTen 16 \\
\hline gelido caldo & gelid hot & A A & hot-cold & itTenTen 16 \\
\hline gelido calore & icy warmth & $\mathrm{AN}$ & hot-cold & itTenTen 16 \\
\hline gelido fuoco & icy fire & $\mathrm{AN}$ & hot-cold & $\begin{array}{l}\text { CORIS + } \\
\text { itTenTen16 }\end{array}$ \\
\hline gelo ardente & burning frost & $\mathrm{NA}$ & hot-cold & itTenTen16 \\
\hline gelo bruciante & burning frost & $\mathrm{NA}$ & hot-cold & itTenTen 16 \\
\hline $\begin{array}{l}\text { ghiaccio } \\
\text { acceso }\end{array}$ & burning ice & $\mathrm{NA}$ & hot-cold & itTenTen 16 \\
\hline $\begin{array}{l}\text { ghiaccio } \\
\text { ardente }\end{array}$ & burning ice & $\mathrm{NA}$ & hot-cold & itTenTen16 \\
\hline $\begin{array}{l}\text { ghiaccio } \\
\text { bollente }\end{array}$ & steaming ice & $\mathrm{N}$ A & hot-cold & $\begin{array}{l}\text { CORIS }+ \\
\text { itTenTen16 }\end{array}$ \\
\hline ghiaccio caldo & hot ice & $\mathrm{NA}$ & hot-cold & $\begin{array}{l}\text { CORIS + } \\
\text { itTenTen16 }\end{array}$ \\
\hline $\begin{array}{l}\text { ghiaccio } \\
\text { cocente }\end{array}$ & searing ice & $\mathrm{NA}$ & hot-cold & itTenTen 16 \\
\hline $\begin{array}{l}\text { ghiaccio } \\
\text { fiammeggiant } \\
\text { e }\end{array}$ & flaming ice & $\mathrm{NA}$ & hot-cold & itTenTen16 \\
\hline $\begin{array}{l}\text { ghiaccio } \\
\text { incandescente }\end{array}$ & $\begin{array}{l}\text { incandescent } \\
\text { ice }\end{array}$ & $\mathrm{NA}$ & hot-cold & itTenTen 16 \\
\hline $\begin{array}{l}\text { ghiaccio } \\
\text { infuocato }\end{array}$ & inflamed ice & $\mathrm{NA}$ & hot-cold & itTenTen 16 \\
\hline $\begin{array}{l}\text { ghiaccio } \\
\text { rovente }\end{array}$ & scorching ice & $\mathrm{NA}$ & hot-cold & itTenTen 16 \\
\hline glaciale calore & glacial warmth & A N & hot-cold & itTenTen 16 \\
\hline $\begin{array}{l}\text { glaciale } \\
\text { fiamma }\end{array}$ & glacial flame & $\mathrm{AN}$ & hot-cold & itTenTen 16 \\
\hline $\begin{array}{l}\text { grandine } \\
\text { incandescente }\end{array}$ & $\begin{array}{l}\text { incandescent } \\
\text { hail }\end{array}$ & $\mathrm{NA}$ & hot-cold & itTenTen 16 \\
\hline $\begin{array}{l}\text { grandine } \\
\text { rovente }\end{array}$ & scorching hail & $\mathrm{NA}$ & hot-cold & itTenTen 16 \\
\hline il fuoco bagna & fire wets (sth) & $\mathrm{S}$ & hot-cold & $\begin{array}{l}\text { CORIS + } \\
\text { itTenTen16 }\end{array}$ \\
\hline $\begin{array}{l}\text { il fuoco } \\
\text { raffredda }\end{array}$ & fire chills (sth) & $\mathrm{S}$ & hot-cold & itTenTen 16 \\
\hline $\begin{array}{l}\text { il gelo } \\
\text { infiamma }\end{array}$ & frost sets fire & $\mathrm{S}$ & hot-cold & itTenTen 16 \\
\hline $\begin{array}{l}\text { il ghiaccio } \\
\text { brucia }\end{array}$ & ice burns & $\mathrm{S}$ & hot-cold & itTenTen 16 \\
\hline infuocato gelo & inflamed frost & A N & hot-cold & itTenTen 16 \\
\hline $\begin{array}{l}\text { infuocato } \\
\text { ghiaccio }\end{array}$ & inflamed ice & $\mathrm{AN}$ & hot-cold & itTenTen 16 \\
\hline $\begin{array}{l}\text { la fiamma } \\
\text { bagna }\end{array}$ & flame wets & $\mathrm{S}$ & hot-cold & itTenTen 16 \\
\hline $\begin{array}{l}\text { la fiamma } \\
\text { gela }\end{array}$ & flame freezes & $\mathrm{S}$ & hot-cold & itTenTen 16 \\
\hline la neve brucia & the snow burns & $\mathrm{S}$ & hot-cold & itTenTen16 \\
\hline $\begin{array}{l}\text { la pioggia } \\
\text { infiamma }\end{array}$ & the rain sets fire & $\mathrm{S}$ & hot-cold & itTenTen 16 \\
\hline neve ardente & burning snow & $\mathrm{N} A$ & hot-cold & $\begin{array}{l}\text { CORIS + } \\
\text { itTenTen16 }\end{array}$ \\
\hline neve bollente & steaming snow & N A & hot-cold & itTenTen 16 \\
\hline neve calda & hot snow & $\mathrm{NA}$ & hot-cold & itTenTen 16 \\
\hline $\begin{array}{l}\text { neve } \\
\text { incandescente }\end{array}$ & $\begin{array}{l}\text { incandescent } \\
\text { snow }\end{array}$ & N A & hot-cold & itTenTen 16 \\
\hline neve infuocata & inflamed snow & N A & hot-cold & itTenTen 16 \\
\hline $\begin{array}{l}\text { pioggia } \\
\text { ardente }\end{array}$ & blazing rain & N A & hot-cold & itTenTen 16 \\
\hline $\begin{array}{l}\text { pioggia } \\
\text { bollente }\end{array}$ & steaming rain & $\mathrm{N} A$ & hot-cold & itTenTen 16 \\
\hline $\begin{array}{l}\text { pioggia } \\
\text { bruciante }\end{array}$ & burning rain & $\mathrm{NA}$ & hot-cold & itTenTen 16 \\
\hline $\begin{array}{l}\text { pioggia } \\
\text { incandescente }\end{array}$ & $\begin{array}{l}\text { incandescent } \\
\text { rain }\end{array}$ & $\mathrm{NA}$ & hot-cold & itTenTen 16 \\
\hline $\begin{array}{l}\text { pioggia } \\
\text { infiammata }\end{array}$ & burning rain & $\mathrm{NA}$ & hot-cold & itTenTen 16 \\
\hline $\begin{array}{l}\text { pioggia } \\
\text { infuocata }\end{array}$ & inflamed rain & N A & hot-cold & $\begin{array}{l}\text { CORIS + } \\
\text { itTenTen16 }\end{array}$ \\
\hline
\end{tabular}

\begin{tabular}{|c|c|c|c|c|}
\hline $\begin{array}{l}\text { pioggia } \\
\text { rovente }\end{array}$ & scorching rain & $\mathrm{NA}$ & hot-cold & itTenTen16 \\
\hline $\begin{array}{l}\text { rovente } \\
\text { ghiaccio }\end{array}$ & scorching ice & AN & hot-cold & itTenTen 16 \\
\hline $\begin{array}{l}\text { giusta } \\
\text { ingiustizia }\end{array}$ & just injustice & A N & $\begin{array}{l}\text { justice- } \\
\text { injustice }\end{array}$ & itTenTen 16 \\
\hline $\begin{array}{l}\text { giustamente } \\
\text { ingiusto }\end{array}$ & justly unjust & Adv A & $\begin{array}{l}\text { justice- } \\
\text { injustice }\end{array}$ & itTenTen 16 \\
\hline $\begin{array}{l}\text { giustizia } \\
\text { ingiusta }\end{array}$ & unjust justice & $\mathrm{NA}$ & $\begin{array}{l}\text { justice- } \\
\text { injustice }\end{array}$ & $\begin{array}{l}\text { CORIS + } \\
\text { itTenTen16 }\end{array}$ \\
\hline $\begin{array}{l}\text { ingiusta } \\
\text { giustizia }\end{array}$ & unjust justice & $\mathrm{AN}$ & $\begin{array}{l}\text { justice- } \\
\text { injustice }\end{array}$ & $\begin{array}{l}\text { CORIS + } \\
\text { itTenTen16 }\end{array}$ \\
\hline $\begin{array}{l}\text { ingiustizia } \\
\text { giusta }\end{array}$ & just unjustice & $\mathrm{NA}$ & $\begin{array}{l}\text { justice- } \\
\text { injustice }\end{array}$ & itTenTen 16 \\
\hline $\begin{array}{l}\text { l'ingiustizia } \\
\text { della giustizia }\end{array}$ & $\begin{array}{l}\text { the injustice of } \\
\text { justice }\end{array}$ & N Prep N & $\begin{array}{l}\text { justice- } \\
\text { injustice }\end{array}$ & itTenTen 16 \\
\hline la morte è vita & death is life & $\mathrm{S}$ & life-death & itTenTen16 \\
\hline la morte vive & death lives & $\mathrm{S}$ & life-death & itTenTen 16 \\
\hline la vita è morte & life is death & $\mathrm{S}$ & life-death & itTenTen 16 \\
\hline $\begin{array}{l}\text { mortalmente } \\
\text { vivo }\end{array}$ & mortally alive & Adv A & life-death & itTenTen 16 \\
\hline morte vitale & vital death & N A & life-death & itTenTen 16 \\
\hline morte viva & living death & $\mathrm{NA}$ & life-death & $\begin{array}{l}\text { CORIS + } \\
\text { itTenTen16 }\end{array}$ \\
\hline morte vivente & living death & $\mathrm{NA}$ & life-death & $\begin{array}{l}\text { CORIS + } \\
\text { itTenTen16 }\end{array}$ \\
\hline morto vivente & living dead & $\mathrm{NA}$ & life-death & $\begin{array}{l}\text { CORIS + } \\
\text { itTenTen16 }\end{array}$ \\
\hline morto vivo & living dead & $\mathrm{NA}$ & life-death & $\begin{array}{l}\text { CORIS + } \\
\text { itTenTen16 }\end{array}$ \\
\hline vita morta & dead life & $\mathrm{NA}$ & life-death & itTenTen 16 \\
\hline vitale morte & vital death & A N & life-death & itTenTen 16 \\
\hline viva morte & living death & A N & life-death & $\begin{array}{l}\text { CORIS + } \\
\text { itTenTen16 }\end{array}$ \\
\hline vivente morte & living death & A N & life-death & itTenTen 16 \\
\hline buia luce & dark light & $\mathrm{AN}$ & light-dark & $\begin{array}{l}\text { CORIS + } \\
\text { itTenTen16 }\end{array}$ \\
\hline buio chiarore & dark gleam & $\mathrm{AN}$ & light-dark & itTenTen 16 \\
\hline buio lucente & shining dark & N A & light-dark & itTenTen 16 \\
\hline buio luminoso & bright dark & $\mathrm{NA}$ & light-dark & $\begin{array}{l}\text { CORIS + } \\
\text { itTenTen16 }\end{array}$ \\
\hline $\begin{array}{l}\text { chiaramente } \\
\text { (o)scuro }\end{array}$ & brightly dark & Adv A & light-dark & itTenTen 16 \\
\hline $\begin{array}{l}\text { chiaramente } \\
\text { buio }\end{array}$ & clearly dark & Adv A & light-dark & itTenTen 16 \\
\hline chiarore scuro & dark gleam & N A & light-dark & itTenTen16 \\
\hline il buio è luce & dark is light & $\mathrm{S}$ & light-dark & itTenTen16 \\
\hline $\begin{array}{l}\text { il buio } \\
\text { illumina }\end{array}$ & $\begin{array}{l}\text { the dark } \\
\text { illuminates } \\
\text { (sth) }\end{array}$ & $\mathrm{S}$ & light-dark & itTenTen 16 \\
\hline $\begin{array}{l}\text { l'oscurità della } \\
\text { luce }\end{array}$ & $\begin{array}{l}\text { the darkness of } \\
\text { the light }\end{array}$ & N Prep N & light-dark & itTenTen16 \\
\hline $\begin{array}{l}\text { l'oscurità } \\
\text { illumina }\end{array}$ & $\begin{array}{l}\text { darkness } \\
\text { illuminates } \\
\text { (sth) }\end{array}$ & $\mathrm{S}$ & light-dark & itTenTen 16 \\
\hline $\begin{array}{l}\text { la luce è } \\
\text { ombra }\end{array}$ & light is shadow & $\mathrm{S}$ & light-dark & itTenTen16 \\
\hline $\begin{array}{l}\text { la luce è } \\
\text { tenebra }\end{array}$ & light is darkness & $\mathrm{S}$ & light-dark & itTenTen16 \\
\hline la luce oscura & $\begin{array}{l}\text { light darkens } \\
\text { (sth) }\end{array}$ & $\mathrm{S}$ & light-dark & itTenTen 16 \\
\hline $\begin{array}{l}\text { la tenebra } \\
\text { della luce }\end{array}$ & $\begin{array}{l}\text { darkness of the } \\
\text { light }\end{array}$ & N Prep N & light-dark & itTenTen 16 \\
\hline $\begin{array}{l}\text { la tenebra è } \\
\text { luce }\end{array}$ & darkness is light & $\mathrm{S}$ & light-dark & itTenTen 16 \\
\hline luce (o)scura & obscure light & $\mathrm{NA}$ & light-dark & $\begin{array}{l}\text { CORIS + } \\
\text { itTenTen16 }\end{array}$ \\
\hline luce buia & dark light & NA & light-dark & $\begin{array}{l}\text { CORIS + } \\
\text { itTenTen16 }\end{array}$ \\
\hline luce oscurante & darkening light & $\mathrm{NA}$ & light-dark & itTenTen16 \\
\hline luce tenebrosa & gloomy light & $\mathrm{NA}$ & light-dark & itTenTen 16 \\
\hline $\begin{array}{l}\text { luminosa } \\
\text { oscurità }\end{array}$ & bright darkness & A N & light-dark & itTenTen 16 \\
\hline $\begin{array}{l}\text { luminosa } \\
\text { tenebra }\end{array}$ & bright shadow & A N & light-dark & itTenTen 16 \\
\hline $\begin{array}{l}\text { luminosament } \\
\text { e oscuro }\end{array}$ & brightly dark & Adv A & light-dark & itTenTen 16 \\
\hline $\begin{array}{l}\text { oscuramente } \\
\text { chiaro }\end{array}$ & darkly clear & Adv A & light-dark & itTenTen 16 \\
\hline $\begin{array}{l}\text { oscuramente } \\
\text { luminoso }\end{array}$ & darkly bright & Adv A & light-dark & itTenTen16 \\
\hline $\begin{array}{l}\text { oscurità } \\
\text { lucente }\end{array}$ & glossy darkness & $\mathrm{NA}$ & light-dark & itTenTen 16 \\
\hline $\begin{array}{l}\text { oscurità } \\
\text { luminosa }\end{array}$ & bright darkness & $\mathrm{NA}$ & light-dark & itTenTen16 \\
\hline $\begin{array}{l}\text { raggiante } \\
\text { oscurità }\end{array}$ & $\begin{array}{l}\text { glowing } \\
\text { darkness }\end{array}$ & $\mathrm{AN}$ & light-dark & itTenTen 16 \\
\hline
\end{tabular}




\begin{tabular}{|c|c|c|c|c|}
\hline $\begin{array}{l}\text { tenebra } \\
\text { lucente }\end{array}$ & glossy shadow & $\mathrm{NA}$ & light-dark & itTenTen 16 \\
\hline $\begin{array}{l}\text { tenebra } \\
\text { luminosa }\end{array}$ & bright shadow & NA & light-dark & $\begin{array}{l}\text { CORIS + } \\
\text { itTenTen16 }\end{array}$ \\
\hline $\begin{array}{l}\text { leggerezza } \\
\text { pesante }\end{array}$ & heavy lightness & $\mathrm{NA}$ & $\begin{array}{l}\text { lightness- } \\
\text { heaviness }\end{array}$ & $\begin{array}{l}\text { CORIS + } \\
\text { itTenTen16 }\end{array}$ \\
\hline $\begin{array}{l}\text { leggermente } \\
\text { pesante }\end{array}$ & lightly heavy & Adv A & $\begin{array}{l}\text { lightness- } \\
\text { heaviness }\end{array}$ & $\begin{array}{l}\text { CORIS + } \\
\text { itTenTen16 }\end{array}$ \\
\hline $\begin{array}{l}\text { pesante } \\
\text { leggerezza }\end{array}$ & heavy lightness & AN & $\begin{array}{l}\text { lightness- } \\
\text { heaviness }\end{array}$ & $\begin{array}{l}\text { CORIS + } \\
\text { itTenTen16 }\end{array}$ \\
\hline amore odiato & hated love & N A & love-hate & itTenTen 16 \\
\hline amore odioso & hateful love & N A & love-hate & itTenTen16 \\
\hline $\begin{array}{l}\text { amorevole } \\
\text { odio }\end{array}$ & loving hate & $\mathrm{AN}$ & love-hate & itTenTen 16 \\
\hline $\begin{array}{l}\text { amorevolment } \\
\text { e odioso }\end{array}$ & lovely hateful & Adv A & love-hate & itTenTen 16 \\
\hline $\begin{array}{l}\text { detestabile } \\
\text { amore }\end{array}$ & awful love & A N & love-hate & CORIS \\
\hline $\begin{array}{l}\text { l'amore } \\
\text { detesta }\end{array}$ & love despises & $\mathrm{S}$ & love-hate & itTenTen 16 \\
\hline l'amore è odio & love is hate & $\mathrm{S}$ & love-hate & itTenTen 16 \\
\hline l'amore odia & love hates & $\mathrm{S}$ & love-hate & itTenTen 16 \\
\hline $\begin{array}{l}\text { l'odio } \\
\text { dell'amore }\end{array}$ & $\begin{array}{l}\text { the hatred of } \\
\text { love }\end{array}$ & N Prep N & love-hate & itTenTen16 \\
\hline l'odio è amore & hate is love & $\mathrm{S}$ & love-hate & itTenTen16 \\
\hline odiato amore & hated love & $\mathrm{AN}$ & love-hate & itTenTen 16 \\
\hline $\begin{array}{l}\text { odio } \\
\text { amar(ti/lo) }\end{array}$ & $\begin{array}{l}\text { I hate to love } \\
\text { you/it }\end{array}$ & $\mathrm{S}$ & love-hate & itTenTen 16 \\
\hline $\begin{array}{l}\text { odio } \\
\text { amorevole }\end{array}$ & loving hate & $\mathrm{NA}$ & love-hate & itTenTen 16 \\
\hline odio amoroso & loving love & $\mathrm{NA}$ & love-hate & $\begin{array}{l}\text { CORIS + } \\
\text { itTenTen16 }\end{array}$ \\
\hline $\begin{array}{l}\text { odiosamente } \\
\text { amabile }\end{array}$ & hatefully lovely & Adv A & love-hate & itTenTen 16 \\
\hline odioso amore & hateful love & AN & love-hate & itTenTen16 \\
\hline $\begin{array}{l}\text { ti amo, odio } \\
\text { mio }\end{array}$ & $\begin{array}{l}\text { I love you, my } \\
\text { hate }\end{array}$ & S & love-hate & itTenTen 16 \\
\hline $\begin{array}{l}\text { ti odio, amore } \\
\text { mio }\end{array}$ & $\begin{array}{l}\text { I hate you, my } \\
\text { love }\end{array}$ & $\mathrm{S}$ & love-hate & itTenTen 16 \\
\hline irreale realtà & unreal reality & $\mathrm{AN}$ & $\begin{array}{l}\text { reality- } \\
\text { unreality }\end{array}$ & $\begin{array}{l}\text { CORIS + } \\
\text { itTenTen16 }\end{array}$ \\
\hline $\begin{array}{l}\text { irrealmente } \\
\text { reale }\end{array}$ & unreally real & Adv A & $\begin{array}{l}\text { reality- } \\
\text { unreality }\end{array}$ & itTenTen 16 \\
\hline irrealtà reale & real unreality & $\mathrm{NA}$ & $\begin{array}{l}\text { reality- } \\
\text { unreality }\end{array}$ & itTenTen 16 \\
\hline $\begin{array}{l}\text { la finzione è } \\
\text { realtà }\end{array}$ & $\begin{array}{l}\text { pretence is } \\
\text { reality }\end{array}$ & $\mathrm{S}$ & $\begin{array}{l}\text { reality- } \\
\text { unreality }\end{array}$ & itTenTen 16 \\
\hline $\begin{array}{l}\text { la irrealtà } \\
\text { della realtà }\end{array}$ & $\begin{array}{l}\text { the unreality of } \\
\text { reality }\end{array}$ & N Prep N & $\begin{array}{l}\text { reality- } \\
\text { unreality }\end{array}$ & itTenTen 16 \\
\hline $\begin{array}{l}\text { la realtà è } \\
\text { finzione }\end{array}$ & $\begin{array}{l}\text { reality is } \\
\text { pretence }\end{array}$ & $\mathrm{S}$ & $\begin{array}{l}\text { reality- } \\
\text { unreality }\end{array}$ & itTenTen 16 \\
\hline $\begin{array}{l}\text { la realtà è } \\
\text { illusione }\end{array}$ & $\begin{array}{l}\text { reality is an } \\
\text { illusion }\end{array}$ & $S$ & $\begin{array}{l}\text { reality- } \\
\text { unreality }\end{array}$ & itTenTen 16 \\
\hline $\begin{array}{l}\text { la realtà è } \\
\text { sogno }\end{array}$ & $\begin{array}{l}\text { reality is a } \\
\text { dream }\end{array}$ & $S$ & $\begin{array}{l}\text { reality- } \\
\text { unreality }\end{array}$ & itTenTen 16 \\
\hline reale irrealtà & real unreality & AN & $\begin{array}{l}\text { reality- } \\
\text { unreality }\end{array}$ & itTenTen 16 \\
\hline $\begin{array}{l}\text { realmente } \\
\text { irreale }\end{array}$ & really unreal & Adv A & $\begin{array}{l}\text { reality- } \\
\text { unreality }\end{array}$ & itTenTen 16 \\
\hline realtà irreale & unreal reality & $\mathrm{NA}$ & $\begin{array}{l}\text { reality- } \\
\text { unreality }\end{array}$ & $\begin{array}{l}\text { CORIS + } \\
\text { itTenTen16 } \\
\end{array}$ \\
\hline $\begin{array}{l}\text { assordante } \\
\text { silenzio }\end{array}$ & $\begin{array}{l}\text { deafening } \\
\text { silence }\end{array}$ & AN & silence-noise & $\begin{array}{l}\text { CORIS + } \\
\text { itTenTen16 }\end{array}$ \\
\hline $\begin{array}{l}\text { bisbigliante } \\
\text { silenzio }\end{array}$ & $\begin{array}{l}\text { whispering } \\
\text { silence }\end{array}$ & $\mathrm{AN}$ & silence-noise & itTenTen 16 \\
\hline boato muto & mute thunder & $\mathrm{NA}$ & silence-noise & itTenTen 16 \\
\hline $\begin{array}{l}\text { eloquente } \\
\text { silenzio }\end{array}$ & eloquent silence & A N & silence-noise & $\begin{array}{l}\text { CORIS + } \\
\text { itTenTen16 }\end{array}$ \\
\hline $\begin{array}{l}\text { eloquentement } \\
\text { e muto }\end{array}$ & eloquently mute & Adv A & silence-noise & itTenTen 16 \\
\hline $\begin{array}{l}\text { eloquentement } \\
\text { e silenzioso }\end{array}$ & eloquently quiet & Adv A & silence-noise & $\begin{array}{l}\text { CORIS + } \\
\text { itTenTen16 }\end{array}$ \\
\hline fragore muto & mute racket & N A & silence-noise & itTenTen 16 \\
\hline $\begin{array}{l}\text { fragore } \\
\text { silenzioso }\end{array}$ & hushed racket & $\mathrm{NA}$ & silence-noise & itTenTen 16 \\
\hline $\begin{array}{l}\text { fragorosament } \\
\text { e silente }\end{array}$ & loudly silent & Adv A & silence-noise & itTenTen 16 \\
\hline $\begin{array}{l}\text { fragorosament } \\
\text { e silenzioso }\end{array}$ & loudly quiet & Adv A & silence-noise & $\begin{array}{l}\text { CORIS + } \\
\text { itTenTen16 }\end{array}$ \\
\hline $\begin{array}{l}\text { fragoroso } \\
\text { silenzio }\end{array}$ & loud silence & A N & silence-noise & $\begin{array}{l}\text { CORIS + } \\
\text { itTenTen16 }\end{array}$ \\
\hline gridare silenzi & $\begin{array}{l}\text { to shout } \\
\text { silences }\end{array}$ & $\mathrm{S}$ & silence-noise & itTenTen 16 \\
\hline $\begin{array}{l}\text { gridare } \\
\text { tacendo }\end{array}$ & $\begin{array}{l}\text { to shout being } \\
\text { silent }\end{array}$ & V V & silence-noise & itTenTen 16 \\
\hline grido muto & mute shout & $\mathrm{NA}$ & silence-noise & $\begin{array}{l}\text { CORIS + } \\
\text { itTenTen16 }\end{array}$ \\
\hline
\end{tabular}

\begin{tabular}{|c|c|c|c|c|}
\hline grido silente & silent shout & $\mathrm{NA}$ & silence-noise & itTenTen 16 \\
\hline $\begin{array}{l}\text { grido } \\
\text { silenzioso }\end{array}$ & quiet shout & $\mathrm{NA}$ & silence-noise & $\begin{array}{l}\text { CORIS + } \\
\text { itTenTen16 }\end{array}$ \\
\hline grido taciuto & silenced shout & $\mathrm{NA}$ & silence-noise & itTenTen 16 \\
\hline $\begin{array}{l}\text { il fragore del } \\
\text { silenzio }\end{array}$ & $\begin{array}{l}\text { the racket of } \\
\text { silence }\end{array}$ & N Prep N & silence-noise & itTenTen 16 \\
\hline $\begin{array}{l}\text { il grido del } \\
\text { silenzio }\end{array}$ & $\begin{array}{l}\text { the shout of } \\
\text { silence }\end{array}$ & N Prep N & silence-noise & itTenTen 16 \\
\hline $\begin{array}{l}\text { il rumore del } \\
\text { silenzio }\end{array}$ & $\begin{array}{l}\text { the noise of } \\
\text { silence }\end{array}$ & N Prep N & silence-noise & itTenTen 16 \\
\hline $\begin{array}{l}\text { il silenzio del } \\
\text { rumore }\end{array}$ & $\begin{array}{l}\text { the silence of } \\
\text { noise }\end{array}$ & N Prep N & silence-noise & itTenTen 16 \\
\hline $\begin{array}{l}\text { il silenzio è } \\
\text { rumore }\end{array}$ & silence is noise & $\mathrm{S}$ & silence-noise & itTenTen 16 \\
\hline $\begin{array}{l}\text { il silenzio è un } \\
\text { grido }\end{array}$ & silence is a cry & $\mathrm{S}$ & silence-noise & itTenTen 16 \\
\hline $\begin{array}{l}\text { il silenzio è un } \\
\text { urlo }\end{array}$ & $\begin{array}{l}\text { silence is a } \\
\text { shout }\end{array}$ & $\mathrm{S}$ & silence-noise & itTenTen16 \\
\hline $\begin{array}{l}\text { il silenzio è } \\
\text { una voce }\end{array}$ & $\begin{array}{l}\text { silence is a } \\
\text { voice }\end{array}$ & $\mathrm{S}$ & silence-noise & itTenTen 16 \\
\hline $\begin{array}{l}\text { il silenzio } \\
\text { grida }\end{array}$ & silence screams & $\mathrm{S}$ & silence-noise & $\begin{array}{l}\text { CORIS + } \\
\text { itTenTen16 }\end{array}$ \\
\hline $\begin{array}{l}\text { il silenzio } \\
\text { mormora }\end{array}$ & $\begin{array}{l}\text { silence } \\
\text { whispers }\end{array}$ & $\mathrm{S}$ & silence-noise & itTenTen 16 \\
\hline $\begin{array}{l}\text { il silenzio nel } \\
\text { rumore }\end{array}$ & $\begin{array}{l}\text { the silence into } \\
\text { the noise }\end{array}$ & N Prep N & silence-noise & itTenTen 16 \\
\hline $\begin{array}{l}\text { il silenzio } \\
\text { parla }\end{array}$ & silence speaks & $\mathrm{S}$ & silence-noise & itTenTen16 \\
\hline $\begin{array}{l}\text { il silenzio } \\
\text { rimbomba }\end{array}$ & silence roars & $\mathrm{S}$ & silence-noise & itTenTen16 \\
\hline $\begin{array}{l}\text { il silenzio } \\
\text { risuona }\end{array}$ & $\begin{array}{l}\text { silence } \\
\text { resounds }\end{array}$ & S & silence-noise & itTenTen16 \\
\hline il silenzio urla & silence cries & $\mathrm{S}$ & silence-noise & itTenTen16 \\
\hline $\begin{array}{l}\text { il suono del } \\
\text { silenzio }\end{array}$ & $\begin{array}{l}\text { the sound of } \\
\text { silence }\end{array}$ & N Prep N & silence-noise & itTenTen 16 \\
\hline $\begin{array}{l}\text { l'urlo di/del } \\
\text { silenzio }\end{array}$ & $\begin{array}{l}\text { the scream of } \\
\text { silence }\end{array}$ & N Prep N & silence-noise & itTenTen 16 \\
\hline $\begin{array}{l}\text { mormorante } \\
\text { silenzio }\end{array}$ & $\begin{array}{l}\text { murmuring } \\
\text { silence }\end{array}$ & $\mathrm{AN}$ & silence-noise & itTenTen16 \\
\hline $\begin{array}{l}\text { mutamente } \\
\text { eloquente }\end{array}$ & $\begin{array}{l}\text { dumbly } \\
\text { eloquent }\end{array}$ & Adv A & silence-noise & itTenTen 16 \\
\hline muto fragore & mute racket & $\mathrm{AN}$ & silence-noise & itTenTen16 \\
\hline muto grido & mute shout & $\mathrm{AN}$ & silence-noise & $\begin{array}{l}\text { CORIS + } \\
\text { itTenTen16 }\end{array}$ \\
\hline muto suono & mute sound & $\mathrm{AN}$ & silence-noise & $\begin{array}{l}\text { CORIS + } \\
\text { itTenTen16 }\end{array}$ \\
\hline muto ululato & mute howling & A N & silence-noise & itTenTen16 \\
\hline muto urlo & mute cry & $\mathrm{AN}$ & silence-noise & itTenTen 16 \\
\hline rumore muto & mute noise & $\mathrm{NA}$ & silence-noise & $\begin{array}{l}\text { CORIS + } \\
\text { itTenTen16 }\end{array}$ \\
\hline $\begin{array}{l}\text { rumore } \\
\text { silenzioso }\end{array}$ & quiet noise & $\mathrm{NA}$ & silence-noise & itTenTen 16 \\
\hline rumore taciuto & silenced noise & $\mathrm{NA}$ & silence-noise & itTenTen 16 \\
\hline $\begin{array}{l}\text { rumorosament } \\
\text { e silenzioso }\end{array}$ & noisily quiet & Adv A & silence-noise & itTenTen16 \\
\hline $\begin{array}{l}\text { rumorosament } \\
\text { e zitto }\end{array}$ & noisily mute & Adv A & silence-noise & itTenTen 16 \\
\hline $\begin{array}{l}\text { rumoroso } \\
\text { silenzio }\end{array}$ & loud silence & $\mathrm{AN}$ & silence-noise & $\begin{array}{l}\text { CORIS + } \\
\text { itTenTen16 }\end{array}$ \\
\hline $\begin{array}{l}\text { silenzio } \\
\text { assordante }\end{array}$ & $\begin{array}{l}\text { deafening } \\
\text { silence }\end{array}$ & $\mathrm{NA}$ & silence-noise & $\begin{array}{l}\text { CORIS + } \\
\text { itTenTen16 }\end{array}$ \\
\hline $\begin{array}{l}\text { silenzio } \\
\text { bisbigliante }\end{array}$ & $\begin{array}{l}\text { whispering } \\
\text { silence }\end{array}$ & $\mathrm{NA}$ & silence-noise & itTenTen 16 \\
\hline $\begin{array}{l}\text { silenzio } \\
\text { eloquente }\end{array}$ & eloquent silence & $\mathrm{NA}$ & silence-noise & $\begin{array}{l}\text { CORIS + } \\
\text { itTenTen16 }\end{array}$ \\
\hline $\begin{array}{l}\text { silenzio } \\
\text { fragoroso }\end{array}$ & loud silence & $\mathrm{NA}$ & silence-noise & $\begin{array}{l}\text { CORIS + } \\
\text { itTenTen16 }\end{array}$ \\
\hline $\begin{array}{l}\text { silenzio } \\
\text { gridato }\end{array}$ & shouted silence & $\mathrm{NA}$ & silence-noise & itTenTen 16 \\
\hline $\begin{array}{l}\text { silenzio } \\
\text { mormorato }\end{array}$ & $\begin{array}{l}\text { murmured } \\
\text { silence }\end{array}$ & N A & silence-noise & itTenTen16 \\
\hline $\begin{array}{l}\text { silenzio } \\
\text { parlato }\end{array}$ & spoken silence & $\mathrm{NA}$ & silence-noise & itTenTen 16 \\
\hline $\begin{array}{l}\text { silenzio } \\
\text { rumoroso }\end{array}$ & noisy silence & $\mathrm{NA}$ & silence-noise & itTenTen 16 \\
\hline $\begin{array}{l}\text { silenzio } \\
\text { sonoro }\end{array}$ & $\begin{array}{l}\text { resounding } \\
\text { silence }\end{array}$ & $\mathrm{NA}$ & silence-noise & itTenTen 16 \\
\hline $\begin{array}{l}\text { silenzio } \\
\text { urlante }\end{array}$ & $\begin{array}{l}\text { screaming } \\
\text { silence }\end{array}$ & $\mathrm{NA}$ & silence-noise & itTenTen 16 \\
\hline silenzio urlato & $\begin{array}{l}\text { screamed } \\
\text { silence }\end{array}$ & $\mathrm{NA}$ & silence-noise & $\begin{array}{l}\text { CORIS + } \\
\text { itTenTen16 }\end{array}$ \\
\hline $\begin{array}{l}\text { silenziosamen } \\
\text { te assordante }\end{array}$ & $\begin{array}{l}\text { quietly } \\
\text { deafening }\end{array}$ & Adv A & silence-noise & itTenTen16 \\
\hline $\begin{array}{l}\text { silenziosamen } \\
\text { te eloquente }\end{array}$ & quietly eloquent & Adv A & silence-noise & itTenTen 16 \\
\hline $\begin{array}{l}\text { silenziosamen } \\
\text { te rumoroso }\end{array}$ & quietly noisy & Adv A & silence-noise & itTenTen 16 \\
\hline
\end{tabular}




\begin{tabular}{|c|c|c|c|c|}
\hline $\begin{array}{l}\text { silenzioso } \\
\text { fracasso }\end{array}$ & silent racket & $\mathrm{AN}$ & silence-noise & itTenTen 16 \\
\hline $\begin{array}{l}\text { silenzioso } \\
\text { fragore }\end{array}$ & silent racket & AN & silence-noise & itTenTen 16 \\
\hline $\begin{array}{l}\text { silenzioso } \\
\text { grido }\end{array}$ & silent shout & A N & silence-noise & $\begin{array}{l}\text { CORIS + } \\
\text { itTenTen16 }\end{array}$ \\
\hline $\begin{array}{l}\text { silenzioso } \\
\text { rumore }\end{array}$ & silent noise & A N & silence-noise & itTenTen 16 \\
\hline $\begin{array}{l}\text { silenzioso } \\
\text { suono }\end{array}$ & silent sound & A N & silence-noise & itTenTen 16 \\
\hline silenzioso urlo & silent scream & AN & silence-noise & itTenTen 16 \\
\hline suono muto & mute sound & N A & silence-noise & itTenTen16 \\
\hline $\begin{array}{l}\text { suono } \\
\text { silenzioso }\end{array}$ & silent sound & $\mathrm{NA}$ & silence-noise & itTenTen 16 \\
\hline suono tacito & tacit sound & N A & silence-noise & itTenTen 16 \\
\hline sussurro muto & mute whisper & $\mathrm{NA}$ & silence-noise & itTenTen 16 \\
\hline $\begin{array}{l}\text { tacitamente } \\
\text { eloquente }\end{array}$ & tacitly eloquent & Adv A & silence-noise & itTenTen 16 \\
\hline tacito grido & tacit shout & $\mathrm{AN}$ & silence-noise & itTenTen16 \\
\hline tacito suono & tacit sound & A N & silence-noise & itTenTen16 \\
\hline taciuto urlo & tacit scream & AN & silence-noise & itTenTen 16 \\
\hline $\begin{array}{l}\text { urlante } \\
\text { silenzio }\end{array}$ & $\begin{array}{l}\text { screaming } \\
\text { silence }\end{array}$ & $\mathrm{AN}$ & silence-noise & itTenTen 16 \\
\hline $\begin{array}{l}\text { urlare (stando) } \\
\text { muto }\end{array}$ & $\begin{array}{l}\text { to scream } \\
\text { (staying) mute }\end{array}$ & V A & silence-noise & itTenTen 16 \\
\hline $\begin{array}{l}\text { urlare (stando) } \\
\text { silenzioso }\end{array}$ & $\begin{array}{l}\text { to scream } \\
\text { (staying) quiet }\end{array}$ & V A & silence-noise & itTenTen 16 \\
\hline $\begin{array}{l}\text { urlare in } \\
\text { silenzio }\end{array}$ & $\begin{array}{l}\text { to scream in } \\
\text { silence }\end{array}$ & V Prep N & silence-noise & itTenTen16 \\
\hline urlare tacendo & $\begin{array}{l}\text { to scream being } \\
\text { silent }\end{array}$ & V V & silence-noise & itTenTen 16 \\
\hline urlato silenzio & $\begin{array}{l}\text { screamed } \\
\text { silence }\end{array}$ & A N & silence-noise & itTenTen 16 \\
\hline urlo muto & mute scream & $\mathrm{NA}$ & silence-noise & $\begin{array}{l}\text { CORIS + } \\
\text { itTenTen16 }\end{array}$ \\
\hline urlo silente & silent scream & N A & silence-noise & itTenTen16 \\
\hline urlo silenzioso & quiet scream & $\mathrm{NA}$ & silence-noise & $\begin{array}{l}\text { CORIS + } \\
\text { itTenTen16 }\end{array}$ \\
\hline voce muta & mute voice & $\mathrm{NA}$ & silence-noise & itTenTen 16 \\
\hline calma veloce & quick calm & $\mathrm{NA}$ & $\begin{array}{l}\text { slowness- } \\
\text { speed }\end{array}$ & itTenTen 16 \\
\hline lenta celerità & slow speed & AN & $\begin{array}{l}\text { slowness- } \\
\text { speed }\end{array}$ & itTenTen 16 \\
\hline lenta rapidità & slow rush & AN & $\begin{array}{l}\text { slowness- } \\
\text { speed }\end{array}$ & itTenTen 16 \\
\hline $\begin{array}{l}\text { lentamente } \\
\text { veloce }\end{array}$ & slowly fast & Adv A & $\begin{array}{l}\text { slowness- } \\
\text { speed }\end{array}$ & itTenTen 16 \\
\hline $\begin{array}{l}\text { lentezza } \\
\text { rapida }\end{array}$ & fast slowness & $\mathrm{NA}$ & $\begin{array}{l}\text { slowness- } \\
\text { speed }\end{array}$ & CORIS \\
\hline $\begin{array}{l}\text { rapida } \\
\text { lentezza }\end{array}$ & $\begin{array}{l}\text { speedy } \\
\text { slowness }\end{array}$ & $\mathrm{AN}$ & $\begin{array}{l}\text { slowness- } \\
\text { speed }\end{array}$ & itTenTen 16 \\
\hline veloce calma & quick calm & $\mathrm{AN}$ & $\begin{array}{l}\text { slowness- } \\
\text { speed }\end{array}$ & itTenTen 16 \\
\hline $\begin{array}{l}\text { veloce } \\
\text { lentezza }\end{array}$ & fast slowness & $\mathrm{AN}$ & $\begin{array}{l}\text { slowness- } \\
\text { speed }\end{array}$ & itTenTen 16 \\
\hline $\begin{array}{l}\text { velocemente } \\
\text { lento }\end{array}$ & quickly slow & Adv A & $\begin{array}{l}\text { slowness- } \\
\text { speed }\end{array}$ & itTenTen 16 \\
\hline acre dolcezza & acrid sweetness & A N & $\begin{array}{l}\text { sweetness- } \\
\text { bitterness }\end{array}$ & itTenTen 16 \\
\hline $\begin{array}{l}\text { amara } \\
\text { dolcezza }\end{array}$ & bitter sweetness & A N & $\begin{array}{l}\text { sweetness- } \\
\text { bitterness }\end{array}$ & $\begin{array}{l}\text { CORIS + } \\
\text { itTenTen16 }\end{array}$ \\
\hline $\begin{array}{l}\text { amaramente } \\
\text { dolce }\end{array}$ & bitterly sweet & Adv A & $\begin{array}{l}\text { sweetness- } \\
\text { bitterness }\end{array}$ & itTenTen 16 \\
\hline aspra dolcezza & sour sweetness & A N & $\begin{array}{l}\text { sweetness- } \\
\text { bitterness }\end{array}$ & itTenTen 16 \\
\hline $\begin{array}{l}\text { dolce } \\
\text { amarezza }\end{array}$ & sweet bitterness & $\mathrm{AN}$ & $\begin{array}{l}\text { sweetness- } \\
\text { bitterness }\end{array}$ & $\begin{array}{l}\text { CORIS + } \\
\text { itTenTen16 }\end{array}$ \\
\hline $\begin{array}{l}\text { dolcemente } \\
\text { acido }\end{array}$ & sweetly acid & Adv A & $\begin{array}{l}\text { sweetness- } \\
\text { bitterness }\end{array}$ & itTenTen 16 \\
\hline $\begin{array}{l}\text { dolcemente } \\
\text { amaro }\end{array}$ & sweetly bitter & Adv A & $\begin{array}{l}\text { sweetness- } \\
\text { bitterness }\end{array}$ & itTenTen 16 \\
\hline $\begin{array}{l}\text { dolcemente } \\
\text { aspro }\end{array}$ & sweetly sour & Adv A & $\begin{array}{l}\text { sweetness- } \\
\text { bitterness }\end{array}$ & itTenTen 16 \\
\hline $\begin{array}{l}\text { dolcezza } \\
\text { amara }\end{array}$ & bitter sweetness & $\mathrm{NA}$ & $\begin{array}{l}\text { sweetness- } \\
\text { bitterness }\end{array}$ & $\begin{array}{l}\text { CORIS + } \\
\text { itTenTen16 }\end{array}$ \\
\hline dolcezza aspra & sour sweetness & N A & $\begin{array}{l}\text { sweetness- } \\
\text { bitterness }\end{array}$ & itTenTen 16 \\
\hline $\begin{array}{l}\text { guerra } \\
\text { pacifica }\end{array}$ & peaceful war & $\mathrm{NA}$ & war-peace & $\begin{array}{l}\text { CORIS + } \\
\text { itTenTen16 }\end{array}$ \\
\hline $\begin{array}{l}\text { inoffensiva } \\
\text { guerra }\end{array}$ & harmless war & A N & war-peace & itTenTen 16 \\
\hline $\begin{array}{l}\text { la guerra è } \\
\text { pace }\end{array}$ & war is peace & $\mathrm{S}$ & war-peace & itTenTen 16 \\
\hline $\begin{array}{l}\text { la pace è } \\
\text { guerra }\end{array}$ & peace is war & $\mathrm{S}$ & war-peace & itTenTen 16 \\
\hline $\begin{array}{l}\text { pacifica } \\
\text { guerra }\end{array}$ & peaceful war & $\mathrm{AN}$ & war-peace & itTenTen 16 \\
\hline
\end{tabular}

\begin{tabular}{lllll}
\hline $\begin{array}{l}\text { la povertà } \\
\text { arricchisce }\end{array}$ & $\begin{array}{l}\text { poverty } \\
\text { enriches }\end{array}$ & $\mathrm{S}$ & $\begin{array}{l}\text { wealth- } \\
\text { poverty }\end{array}$ & itTenTen16 \\
\hline $\begin{array}{l}\text { la povertà è } \\
\text { ricchezza }\end{array}$ & $\begin{array}{l}\text { poverty is } \\
\text { wealth }\end{array}$ & $\mathrm{S}$ & $\begin{array}{l}\text { wealth- } \\
\text { poverty }\end{array}$ & itTenTen16 \\
\hline $\begin{array}{l}\text { la ricchezza } \\
\text { della povertà }\end{array}$ & $\begin{array}{l}\text { the wealth of } \\
\text { poverty }\end{array}$ & $\mathrm{N}$ Prep N & $\begin{array}{l}\text { wealth- } \\
\text { poverty }\end{array}$ & itTenTen16 \\
\hline $\begin{array}{l}\text { la ricchezza } \\
\text { impoverisce }\end{array}$ & $\begin{array}{l}\text { wealth } \\
\text { impoverishes }\end{array}$ & $\mathrm{S}$ & $\begin{array}{l}\text { wealth- } \\
\text { poverty }\end{array}$ & itTenTen16 \\
\hline $\begin{array}{l}\text { povera } \\
\text { ricchezza }\end{array}$ & poor wealth & $\mathrm{A} \mathrm{N}$ & $\begin{array}{l}\text { wealth- } \\
\text { poverty }\end{array}$ & $\begin{array}{l}\text { CORIS + } \\
\text { itTenTen16 }\end{array}$ \\
\hline $\begin{array}{l}\text { poveramente } \\
\text { ricco }\end{array}$ & poorly rich & Adv A & $\begin{array}{l}\text { wealth- } \\
\text { poverty }\end{array}$ & itTenTen16 \\
\hline povertà ricca & rich poverty & N A & $\begin{array}{l}\text { wealth- } \\
\text { poverty }\end{array}$ & itTenTen16 \\
\hline $\begin{array}{l}\text { ricca povertà } \\
\text { rich poverty }\end{array}$ & A N & $\begin{array}{l}\text { wealth- } \\
\text { poverty }\end{array}$ & itTenTen16 \\
\hline $\begin{array}{l}\text { riccamente } \\
\text { povero }\end{array}$ & richly poor & Adv A & $\begin{array}{l}\text { wealth- } \\
\text { poverty }\end{array}$ & itTenTen16 \\
\hline $\begin{array}{l}\text { ricchezza } \\
\text { povera }\end{array}$ & poor wealth & N A & $\begin{array}{l}\text { wealth- } \\
\text { poverty }\end{array}$ & itTenTen16 \\
\hline
\end{tabular}

